\title{
Support of Decision-Making under Risk by a Risk-Averse Decision-Maker
}

\author{
Andrzej Łodziński*
}

\begin{abstract}
The paper presents a method of supporting decision-making under risk by a risk-averse decision-maker. Decision-making under risk occurs when the outcome of the system is ambiguous and depends on the state of the environment. The problem is considered as a multi-criteria optimization. The decision support method consists of interactive conduct of the process of decision-making. The decision is made by means of solving a problem with controlling parameters, which determine the aspirations of the decision-maker and evaluating the obtained solutions. The decision-maker sets parameters for which a solution is determined. Subsequently, he or she assesses the obtained solution, accepting or rejecting it. In the latter case, the decision-maker sets new values for the parameters and the problem is solved again. The present paper presents a discrete example of support for decision making under risk.
\end{abstract}

Keywords: decision-making under risk, multi-criteria optimization, equitably efficient solution, scalarizing function, decision support system

Mathematics Subject Classification: 90B50, 68U35, 91B06

Submitted: September 12, 2019

Revised: December 30, 2019

(C) 2019 Author. This is an open access publication, which can be used, distributed and reproduced in any medium according to the Creative Commons CC-BY 4.0 License. License requiring that the original work has been properly cited.

\section{INTRODUCTION}

The paper presents a method of supporting decision-making under risk by a risk-averse decision-maker. Decision-making under risk occurs when the results of actions taken by the decision-maker are random, as unexpected circumstances or confounders may occur. These unexpected circumstances or confounders (conditions of the surroundings), called scenarios, are caused by factors which are beyond the control of the decision-maker but which exert a significant impact on the results of the decision. At the same time, each scenario clearly defines the implementation of the results of individual decisions. Each action leads to one of the specified set of results. The decision-maker cannot determine

\footnotetext{
* Warsaw University of Life Sciences, Faculty of Applied Informatics and Mathematics, Warsaw, Poland, e-mail: andrzej_lodzinski@sggw.pl
} 
with absolute certainty to which result each action will lead, but he or she can calculate the probability that a given result will occur. Thus, the result of each decision is random variable (Luce, Raiffa, 1996; Ogryczak, 2002; Taghavi-Fard et al., 2009).

Decision-making under risk by a risk-averse decision-maker is modeled with a special problem of multi-criteria optimization, i.e., simultaneous maximizing of a vector function, whose particular coordinates represent the result of the decision in which the given scenario occurs. This problem involves functions constituting the cumulative values of ordered evaluation vectors. Multi-criteria optimization methods do not yield one solution which the decision-maker should be able to choose. The decision support method consists of conducting the process of decision-making interactively, i.e. a decision is made by solving a problem with controlling parameters which determine the aspirations of the decision-maker, and evaluating the obtained solutions. The decision-maker sets a parameter for which a solution is determined. Subsequently, he or she assesses the obtained solution, accepting or rejecting it. In the latter case, the decision-maker sets new values for the parameters and the problem is solved again. The decision-making process is not a one-time procedure, but an iterative process in which the decision-maker learns about a decision problem.

\section{MODELING DECISION-MAKING UNDER RISK BY A RISK-AVERSE DECISION-MAKER}

The problem of decision-making under risk is modeled by introducing scenarios which represent possible conditions of the surroundings. A probability distribution is provided for these scenarios. If it is assumed that the probabilities of the occurrence of individual scenarios are rational numbers, repeating the relevant scenarios may lead to a situation where the probability of the occurrence of each scenario is the same. For example, the selection between random variables $Y^{\prime}$ and $Y^{\prime \prime}$ :

$$
P\left(Y^{\prime}=x\right)=\left\{\begin{array}{l}
\frac{1}{2} x=3 \\
\frac{1}{2} x=4
\end{array} \quad \text { and } \quad P\left(Y^{\prime \prime}=x\right)=\left\{\begin{array}{l}
\frac{1}{4} x=2 \\
\frac{3}{4} x=5
\end{array}\right.\right.
$$

is equivalent to the problem of choosing between two lotteries $y^{\prime}=(3,2,4,4)$ and $y^{\prime \prime}=(2,5,5,5)$ with equally probable outcomes, where the order of outcomes is not important.

The number of occurrences of a given scenario corresponds to the probability attributed to it. The specific scenarios $S_{i}, i=1, \ldots, m$ correspond to the appropriate realization of the evaluation function $f_{i}(x), i=1, \ldots, m$. A higher value of the evaluation function is preferred in each scenario.

There are situations in which one of $m$ possible results of $f_{1}(x), \ldots, f_{m}(x)$ occurs for each decision $x \in X_{0}$. The probabilities of these results are equal and are represented by $p=1 m$. 
The problem of decision-making under risk by a risk-averse decision-maker is considered as a multi-criteria optimization problem:

$$
\max _{x}\left\{\left(f_{1}(x), \ldots, f_{m}(x)\right): x \in X_{0}\right\}
$$

where:

$x$ - a vector of decision variables, belonging to the set of feasible decisions; $x \in X_{0}$,

$x_{0}$ - scenarios (conditions of the surroundings); $x_{0} \subset R^{n}, S_{i}, i=1, \ldots, m$,

$f$ - a vector function $f=\left(f_{1}, \ldots, f_{m}\right)$ which attributes an evaluation vector $y_{i}=$ $f_{i}(x)$ to each decision variable vector $x \in X_{0}$; individual coordinates $y_{i}=f_{i}(x)$ represent scalar evaluation functions, i.e., a decision $x$ when the scenario $S_{i}$, $i=1, \ldots, m$ occurs,

$X_{0}$ - the set of feasible decisions; $X_{0} \subset R^{n}$.

This is a problem of multi-criteria optimization reduced to equally probable scenarios. The results are equally probable i.e., each coordinate of the evaluation function has the same weight (Ogryczak, 2002). The problem of multi-criteria optimization (1) is one with homogeneous assessments whose maximization of all functions is equally important. Individual assessments, although generated by different functions, are expressed on the same scale, which enables the comparison of their values.

Vector function $y=f(x)$ assigns to each decision variable vector $x$ an evaluation vector $y \in Y_{0}$, which measures the quality of decision $x$ from the point of view of all quality indicators $y_{1}, \ldots, y_{m}$. The image of feasible set $X_{0}$ for function $f$ is a set of obtainable evaluation vectors $Y_{0}$.

The problem is considered in the evaluation space, i.e., the following problem is considered:

$$
\max _{x}\left(y_{1}, \ldots, y_{m}\right): x \in X_{0}
$$

where:

$x$ - a decision variable vector; $x \in X_{0}$,

$y$ - an evaluation vector $y=\left(y_{1}, \ldots, y_{m}\right)$, particular coordinates $y_{i}$ represent the result of a decision $x$ in the scenario $S_{i}, i=1, \ldots, m ; Y_{0}$ is the set of obtainable evaluation vectors.

Evaluation vector $y=\left(y_{1}, \ldots, y_{m}\right)$ in a multi-criteria problem (2) represents the result of a decision $x$ in the form of a vector with $m$ equally probable $p=1 / m$ coordinates $y_{i}, i=1, \ldots, m$.

\section{EQUITABLY EFFICIENT SOLUTION}

The decision-maker whose utility is increasing at a decreasing rate is characterized by risk aversion. He attaches greater importance to improving the value of smaller ones. Decision-making under risk by a risk-averse decision-maker modeled as a special problem of multi-criteria optimization with a preference relation should additionally satisfy the requirements of anonymity and the Pigou-Dalton transfer principle. The solution of this kind of problem of multi-criteria optimization is an equitably efficient decision. 
Nondominated solutions (optimum Pareto) are defined as follows:

$$
\left.\hat{Y}_{0}=\left\{\hat{y} \in Y_{0}:(\hat{y}+\tilde{D}) \cap Y_{0}=\emptyset\right)\right\}
$$

where $\tilde{D}=D \backslash\{0\}$ is a positive cone without a top.

The following can be assumed as a positive cone: $\tilde{D}=R_{+}^{m}$ (Lewandowski and Wierzbicki, 1989; Wierzbicki et al., 2000).

The decision $\hat{x} \in X_{0}$ is referred to as an efficient (Pareto optimal) decision if the corresponding evaluation vector $\hat{y}=f(\hat{x})$ is a nondominated vector.

In multi-criterial problem (1), which is used to make decisions under risk by a risk averse decision-maker, the preference relation should additionally satisfy the requirements of anonymity and the transfer principle.

In problem (2), the decision-making preference relationship should be impartial due to individual assessment functions. This requirement is mathematically formalized as a property of the anonymity of the preference relation.

A relation is called an anonymous relation when the following property occurs for each evaluation vector $y=\left(y_{1}, y_{2}, \ldots, y_{m}\right) \in R^{m}$ and any permutation $P$ of set $1, \ldots, m$ :

$$
\left(y_{P(1)}, y_{P(2)}, \ldots, y_{P(m)}\right) \approx\left(y_{1}, y_{2}, \ldots, y_{m}\right)
$$

No distinction is made between results that differ in the order of coordinates. Evaluation vectors with the same coordinates, but in different orders, are identical.

Moreover, the preference model which is used to make decisions under risk by a risk averse decision-maker should satisfy the transfer principle, which states that, given two vectors with the same coordinate sum, the vector with a smaller divergence of assessments is preferred for a decision-maker characterized by risk aversion. The principle is mathematically formulated as follows: for evaluation vector $y=\left(y_{1}, y_{2}, \ldots, y_{m}\right) \in R^{m}$ :

$$
y_{i^{\prime}}>y_{i^{\prime \prime}} \Rightarrow y-\varepsilon \cdot e_{i^{\prime}}+\varepsilon \cdot e_{i^{\prime \prime}} \succ y \quad \text { for } \quad 0<y_{i^{\prime \prime}}-y_{i^{\prime}}<\varepsilon
$$

This principle states that the transfer of a small amount from the superior evaluation vector coordinate along with a simultaneous improvement of the inferior coordinate by the same value yields an evaluation vector that is strictly preferred in relation to the source evaluation vector; i.e., given two vectors with the same coordinate sum, a vector with a smaller divergence of assessments is preferred for a decision-maker characterized by risk aversion. The corrected evaluation vector is preferable to the original vector, as there is greater certainty that an average value will be achieved. This is a structure of risk reduction.

The nondominated vector satisfying the property of anonymity and the principle transfers is referred to as an equitably nondominated vector. A set of equitably nondominated vectors is determined as $\hat{Y}_{0 e}$. In the decision space, an equitably efficient decision is determined. Decision $\hat{x} \in X_{0}$ is an equitably efficient decision when the corresponding evaluation vector $\hat{y}=f(\hat{x})$ is an equitably nondominated vector. A set of equitably efficient decisions is determined as $\hat{X}_{0 e}$. 
A decision-maker with equitable dominance preferences is characterized by an aversion to risk.

The equitable dominance relation can be expressed as a relation of inequality for cumulative evaluation vectors placed in order. The relation can be written as the transformation $\bar{T}: R^{m} \rightarrow R^{m}$, which cumulates the coordinates of an evaluation vector placed in nonincreasing order.

Transformation $\bar{T}: R^{m} \rightarrow R^{m}$ is determined as follows:

$$
\bar{T}_{i}(y)=\sum_{l=1}^{i} T_{i}(y) \quad \text { dla } \quad i=1,2, \ldots, m
$$

where: $T(y)$ is a vector with nonincreasing ordered coordinates of the vector $y$, i.e. $T(y)=\left(T_{1}(y), T_{2}(y), \ldots, T_{m}(y)\right)$, where $T_{1}(y) \leqslant T_{2}(y) \leqslant \ldots \leqslant T_{m}(y)$ and there is permutation $P$ of set $\{1, \ldots, m\}$ such that $T_{i}(y)=y_{P(i)}$ for $i, \ldots, m$.

Equitable dominance relation $\succ_{e}$ is an ordinary vector-valued dominance for vectors with coordinates which are cumulative values of the evaluation vector placed in order (Yager, 1988, Kostreva et al., 2005; Ogryczak et al., 2008).

Evaluation vector $y^{1}$ equitably dominates vector $y^{2}$ if the following condition is satisfied:

$$
y^{1} \succ_{e} y^{2} \Leftrightarrow \bar{T}\left(y^{1}\right) \geqslant \bar{T}\left(y^{2}\right)
$$

Inequality (7) is consistent with the relation second stochastic of dominance (SSD).

The decision-maker compares and contrasts decisions according to second-order dominance. The dominant distribution according to SSD ensures a higher expected value for each increasing, concave usability function.

The solution of a decision problem consists of the determination of an equitably efficient decision corresponding to the decision-maker's preferences.

\section{TECHNIQUE OF EQUITABLY EFFICIENT DECISION GENERALIZATION}

Equitably efficient decisions for a multiple-criteria optimization problem (1) are obtained by solving a special multi-criteria optimization problem with the function $\bar{T}(y)$, i.e. a problem with the function of cumulated ordered coordinates of assessment vectors. The problem is as follows:

$$
\max _{y}\left\{\left(\bar{T}_{1}, \bar{T}_{2}(y), \ldots, \bar{T}_{m}(y)\right): y \in Y_{0}\right\}
$$

where:

$y$ - an evaluation vector; $y=\left(y_{1}, y_{2}, \ldots, y_{m}\right)$,

$\bar{T}(y)$ - a cumulative evaluation vector placed in order, $Y_{0}$ is a set of obtainable evaluation vectors; $\bar{T}(y)=\left(\bar{T}_{1}(y), \bar{T}_{2}(y), \ldots, \bar{T}_{m}(y)\right)$.

An efficient solution to multi-criteria optimization problem (8) is an equitably efficient solution to multi-objective problem (1). 
Scalarization of multi-objective problem (8) is solved with the scalarizing function $s: Y \times \Omega \Rightarrow R^{1}$ :

$$
\max _{x}\left\{s(y, \bar{y}): x \in X_{0}\right\}
$$

where:

$y$ - an evaluation vector; $y=\left(y_{1}, y_{2}, \ldots, y_{m}\right)$,

$\bar{y}$ - controlling parameters for individual evaluations; $\bar{y}=\left(\bar{y}_{1}, \bar{y}_{2}, \ldots, \bar{y}_{m}\right)$.

This is a problem of the single-objective optimization of an especially created scalarizing function of two variables - evaluation vector $y \in Y$ and controlling parameter $\bar{y} \in \Omega \subset R^{m}$ with real value, i.e. function $s: Y \times \Omega \Rightarrow R^{1}$. Parameter $\bar{y}=\left(\bar{y}_{1}, \bar{y}_{2}, \ldots, \bar{y}_{m}\right)$ is at the disposal of the decision-maker, which enables him or her to browse a set of equitably efficient solutions.

The complete and sufficient parametrization of the set of equitably efficient solutions $\hat{Y}_{0 e}$ can be achieved, using the method of the reference point for problem (8). The method uses aspiration levels as controlling parameters. Aspiration levels are evaluation function values that satisfy the decision-maker.

The scalarizing function in the reference point method takes the following form:

$$
s(y, \bar{y})=\min _{1 \leqslant i \leqslant m}\left(\bar{T}_{i}(y)-\bar{T}_{i}(\bar{y})_{i}\right)+\varepsilon \cdot \sum_{i=1}^{m}\left(\bar{T}_{i}(y)-\bar{T}_{i}\left(\bar{y}_{i}\right)\right.
$$

where:

$$
\begin{aligned}
y- & \text { an evaluation vector; } y=\left(y_{1}, y_{2}, \ldots, y_{m}\right), \\
\bar{T}(y)- & \text { a cumulative evaluation vector placed in order; } \bar{T}(y)=\left(\bar{T}_{1}(y), \bar{T}_{2}(y), \ldots,\right. \\
& \left.\bar{T}_{m}(y)\right), \\
\bar{y}- & \text { an aspiration level vector; } \bar{y}=\left(\bar{y}_{1}, \bar{y}_{2}, \ldots, \bar{y}_{m}\right), \\
T(\bar{y})- & \text { a cumulative aspiration level vector placed in order; } T(\bar{y})=\left(T_{1}(\bar{y}), T_{2}(\bar{y}), \ldots,\right. \\
& \left.T_{m}(\bar{y})\right), \\
\varepsilon- & \text { an arbitrarily small, positive regularization parameter. }
\end{aligned}
$$

This kind of scalarizing function, called an achievement function, measures the proximity of a given solution to the aspiration level. The decision-maker strives to arrive at a solution that comes as close as possible to the fulfilment of the specified requirements, i.e., aspiration levels (Lewandowski and Wierzbicki, 1989; Wierzbicki et al., 2000, Ogryczak, 2002).

The maximum of such a function due to $x$ determines equitably efficient solution $\hat{y}$ and the equitably efficient decision $\hat{x}$ which generates it. The equitably efficient solution $\hat{x}$ is determined depending on the values of aspiration levels $\bar{y}$.

\section{METHOD FOR THE SELECTION OF AN EQUITABLY EFFICIENT DECISION}

The solution to the problem of multi-criteria optimization comprises an entire set of solutions, thus the decision-maker should make such a decision with the use an interactive computer system, which enables a controlled review of a set of solutions. 
On the basis of the values of controlling parameters provided by the decision-maker, the problem is solved and the system presents a solution corresponding to the current values of these parameters for analysis.

Function (10) is a tool for searching the set of solutions. The maximum of this function depends on the parameter which the decision-maker uses to select the solution. In the reference point method, the decision-maker expresses his or her preferences by identifying the value $\bar{y}_{i}, i=1, \ldots, m$ that would fully satisfy them for each function. These values constitute the aspiration level for a given evaluation function. The controlling parameter in the form of aspiration levels represents real values that the decision-maker can readily understand and which characterize their preferences. Aspiration levels are expressed in terms of the values of individual evaluation functions.

The decision support method is an iterative method consisting of the alternate execution of the following:

- calculations which provide further equitably efficient solutions;

- dialog with the decision-maker, which is a source of additional information about his or her preferences.

The decision support method is presented in Figure 1.

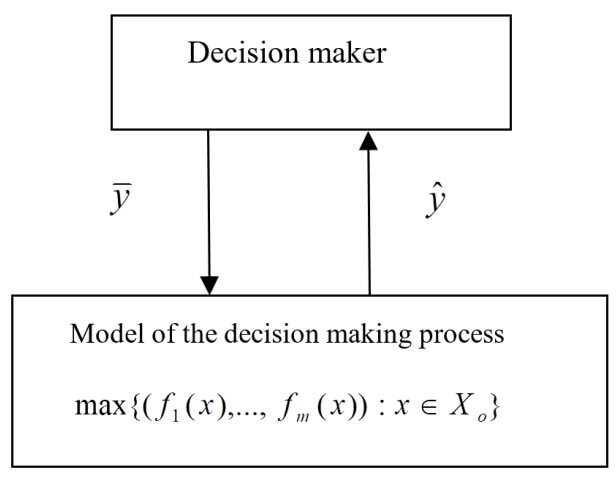

Fig. 1. Decision support method

This decision support method does not force the decision-maker into any rigid scenario for the analysis of the decision problem and, moreover, it enables him or her to modify his or her preferences during problem analysis. In this kind of decision-making support, the decision-maker plays the central role.

\section{EXAMPLE - DECISION SUPPORT UNDER RISK CONDITIONS}

To illustrate the support of a decision made under risk by a risk-averse decision-maker, a simple discrete example of the selection of one of eight decisions in the case of three scenarios is presented In Table 1 . The probabilities of the individual scenarios are as follows: $P_{1}=0.3, P_{2}=0.5$ and $P_{3}=0.2$. 
Table 1. Decision results for individual scenarios

\begin{tabular}{|c|c|c|c|}
\hline Decisions & $S_{1}$ & $S_{2}$ & $S_{3}$ \\
\hline$x_{1}$ & 48 & 59 & 60 \\
\hline$x_{2}$ & 40 & 52 & 58 \\
\hline$x_{3}$ & 46 & 48 & 61 \\
\hline$x_{4}$ & 47 & 63 & 57 \\
\hline$x_{5}$ & 42 & 54 & 55 \\
\hline$x_{6}$ & 41 & 53 & 60 \\
\hline$x_{7}$ & 49 & 64 & 52 \\
\hline$x_{8}$ & 46 & 60 & 56 \\
\hline
\end{tabular}

As the applicable configuration of conditions is unknown, the problem is a choice under risk.

The decision problem assumes the form of a multi-criteria optimization problem:

$$
\max _{x}\left\{y^{1}, y^{2}, y^{3}, y^{4}, y^{5}, y^{6}, y^{7}, y^{8} x \in\left\{x_{1}, \ldots, x_{8}\right\}\right\}
$$

where the results of each decisions are the following vectors:

$$
\begin{aligned}
& y^{1}=(48,59,60) \text { for decision } x_{1} \\
& y^{2}=(40,52,58) \text { for decision } x_{2} \\
& y^{3}=(46,48,61) \text { for decision } x_{3} \\
& y^{4}=(47,63,57) \text { for decision } x_{4} \\
& y^{5}=(42,54,55) \text { for decision } x_{5} \\
& y^{6}=(41,53,60) \text { for decision } x_{6} \\
& y^{7}=(49,64,52) \text { for decision } x_{7} \\
& y^{8}=(46,60,56) \text { for decision } x_{8}
\end{aligned}
$$

where individual coordinates of the evaluation vector occur with probabilities $P_{1}=0.3$, $P_{2}=0.5$ and $P_{3}=0.2$.

The problem consists of selecting a decision for which the evaluation vector is maximal in terms of the equitable dominance relation.

Repeating the appropriate scenarios results in a situation in which the probability of each scenario is the same: $p=1 / 10$. The problem obtained is equivalent to the source problem, where results for each decision $x \in\left\{x_{1}, \ldots, x_{8}\right\}$ are the following evaluation vectors with coordinates that are equally probable:

$$
\begin{aligned}
& y^{1}=(48,48,48,59,59,59,59,59,60,60) \\
& y^{2}=(40,40,52,52,52,52,52,52,58,58) \\
& y^{3}=(46,46,46,48,48,48,48,48,61,61) \\
& y^{4}=(47,47,47,63,63,63,63,63,57,57) \\
& y^{5}=(42,42,42,54,54,54,54,54,55,55) \\
& y^{6}=(41,41,41,53,53,53,53,53,60,60) \\
& y^{7}=(49,49,49,64,64,64,64,64,52,52) \\
& y^{8}=(46,46,46,60,60,60,60,60,56,56)
\end{aligned}
$$


To enable the comparison of vectors in terms of equitable dominance, cumulative vector coordinates placed in order are ordered and the following evaluation vectors for each decision are obtained:

$$
\begin{aligned}
& \bar{T}\left(y^{1}\right)=(48,96,144,203,262,321,380,439,499,599) \\
& \bar{T}\left(y^{2}\right)=(40,80,120,172,224,276,328,380,438,496) \\
& \bar{T}\left(y^{3}\right)=(46,92,138,186,234,282,330,378,439,550) \\
& \bar{T}\left(y^{4}\right)=(47,94,141,198,225,318,381,444,507,570) \\
& \bar{T}\left(y^{5}\right)=(42,84,126,180,234,288,342,396,451,506) \\
& \bar{T}\left(y^{6}\right)=(41,82,123,176,229,282,335,388,448,508) \\
& \bar{T}\left(y^{7}\right)=(49,98,147,199,251,315,379,443,507,571) \\
& \bar{T}\left(y^{8}\right)=(46,92,138,194,250,310,370,430,490,550)
\end{aligned}
$$

The set of equitable non-dominated vectors is as follows: $\hat{Y}_{0 e}=\left\{y^{1}, y^{4}, y^{7}\right\}$. Three decisions, i.e.: $x_{1}, x_{4}$ and $x_{7}$, are equitably efficient decisions. When a choice is being made one of them is selected and the other decisions rejected regardless of individual preferences. These three decisions are not comparable in terms of equitable preference relation. The choice between them depends on the decision-maker's individual preferences.

To determine equitably efficient solutions to problem (10), the reference point method is used for the problem with the cumulative coordinates of evaluation vector placed in order. The decision-maker controls decision making by providing the desired value for the aspiration level for each scenario:

$$
\bar{y}=\left(\bar{y}_{1}, \bar{y}_{1}, \bar{y}_{1}, \bar{y}_{2}, \bar{y}_{2}, \bar{y}_{2}, \bar{y}_{3}, \bar{y}_{3}, \bar{y}_{3}\right)
$$

where:

$\bar{y}_{1}$ - the value of aspiration level for the first scenario,

$\bar{y}_{2}$ - the value of aspiration level for the second scenario,

$\bar{y}_{3}$ - is the value of aspiration level for the third scenario.

The course of multi-objective analysis is presented in Table 2.

Table 2. Interactive search for a satisfactory solution (source: the author's own calculations)

\begin{tabular}{|l|l|}
\hline \multicolumn{1}{|c|}{ Iteration } & \multicolumn{1}{c|}{ Decision } \\
\hline $\begin{array}{l}\text { 1. Aspiration level } \bar{y} \\
\text { Solution } \hat{x}\end{array}$ & $\begin{array}{l}\bar{y}=(49,49,49,64,64,64,64,61,61) \\
\text { decision } x_{7}\end{array}$ \\
\hline $\begin{array}{l}\text { 2. Aspiration level } \bar{y} \\
\text { Solution } \hat{x}\end{array}$ & $\bar{y}=(45,45,45,62,62,62,62,58,58)$ \\
& decision $x_{x}$ \\
\hline 3. Aspiration level $\bar{y}$ & $\bar{y}=(46,46,46,61,61,61,61,57,57)$ \\
Solution $\hat{x}$ & decision $x_{1}$ \\
\hline 4. Aspiration level $\bar{y}$ & $\bar{y}=(43,43,43,60,60,60,60,55,55)$ \\
Solution $\hat{x}$ & decision $x_{7}$ \\
\hline 5. Aspiration level $\bar{y}$ & $\bar{y}=(44,44,44,59,59,59,59,58,58)$ \\
Solution $\hat{x}$ & decision $x_{1}$ \\
\hline
\end{tabular}


At the beginning of the selection, the decision-maker identifies the aspiration levels as the optimal values that can be obtained separately for each scenario and changes the aspiration levels in subsequent iterations depending on his or her preferences. In the first iteration, the decision-maker determines his or her preferences as the aspiration level equaling vector $\bar{y}=(49,49,49,64,64,64,64,61,61)$ and obtains $x_{7}$ as the solution. In the second iteration, the decision-maker lowers the requirements for all scenarios, sets vector $\bar{y}=(45,45,45,62,62,62,62,58,58)$ as the aspiration level and obtains $x_{4}$ as the solution. In the third iteration, the decision-maker raises the requirements for the first scenario, lowers the requirements for the second and third scenarios, sets vector $\bar{y}=(46,46,46,61,61,61,61,57,57)$ as the aspiration level and obtains $x_{1}$ as the solution.

In the fourth iteration, the decision-maker lowers the requirements for all scenarios, sets vector $\bar{y}=(43,43,43,60,60,60,60,55,55)$ as the aspiration level and obtains $x_{7}$ as the solution. In the fifth iteration, the decision-maker raises the requirements for the first and third scenarios, lowers the requirements for the second scenario, sets vector $\bar{y}=(44,44,44,59,59,59,59,58,58)$ as the aspiration level and obtains $x_{1}$ as the solution.

The decision support method enables the decision-maker to choose any equitably efficient solution. The final selection of the specific solution depends on the decisionmaker's preferences. The presented example shows that this method enables the decisionmaker to become acquainted with his or her decision-making capacity in the course of interactive analysis and a search for a satisfying solution.

\section{CONCLUSIONS}

The paper presents a method for decision support under risk by a risk-averse decision-maker. The decision is made by solving the problem of multi-criteria optimization. The method is characterized by the following:

- The use of aspiration points and optimality of the achievement function to organize interaction with the decision-maker;

- The assumption that the decision-maker's preferences are not fully shaped and thus change during the decision making process, and that the main task of the method is to support the learning of the decision-maker about the decision problem rather than the final act of choice.

The method provides an entire set of solutions of equitably efficient decisions and enables the decision-maker to make a free choice. Such a procedure does not replace the decision-maker; rather, the entire decision-making process is controlled by the decision-maker.

\section{REFERENCES}

Kostreva, M., Ogryczak, W., Wierzbicki, A.P., 2005. Equitable Aggregation and Multiple Criteria Analysis. European Journal of Operational Research, 158, pp. 362-377.

Lewandowski, A., Wierzbicki, A.P. (eds.), 1989. Aspiration Based Decision Support Systems. Lecture Notes in Economics and Mathematical Systems, 331, Springer-Verlag, Berlin Heidelberg. 
Luce, D., Raiffa, H., 1964. Gry i decyzje. PWN, Warszawa.

Ogryczak, W., 2002. Multicriteria Optimization and Decisions under Risk. Control and Cybernetics, 31(4), pp. 975-1003.

Ogryczak, W., Wierzbicki, A.P., Milewski, M., 2008. A Multi-Criteria Approach to Fair and Efficient Bandwidth Allocation. Omega, 36, pp. 45-463.

Taghavi-Fard, M.T., Khalili-Damghani, K., Tavakkoli Moghaddam, R., 2009. Decision Making under Uncertain and Risky Situations. Society of Actuaries, pp. 1-31.

Wierzbicki, A.P., Makowski, N., Wessels, J. (eds.), 2000. Model-based Decision Support Methodology with Environmental Applications. IIASA Kluwer, Laxenburg Dordrecht.

Yager, R., 1988. On ordered weighted averaging aggregation operators in multi-criteria decision making. IEEE Transactions on Systems, Man, and Cybernetics. Volume 18, pp. 183-190. 